\title{
Misure di associazione basate sulla differenza relativa del rischio: rischio relativo e odds ratio
}

\author{
Francesco Franco ${ }^{1}$, Anteo Di Napoli ${ }^{2}$ \\ ${ }^{1}$ Regione Lazio, Roma \\ ${ }^{2}$ Comitato Tecnico-Scientifico RIDT (Registro Italiano Dialisi e Trapianto), Roma
}

\begin{abstract}
Measures of association based on relative differences in risk: relative risk and odds ratio
To assess the relationship between exposure and outcome is the main purpose of epidemiological studies. The measures of association evaluate the direction and magnitude of this relationship. Relative risk (RR) and odds ratio (OR) are the measures used most when the strength of the association between an exposure and an outcome is measured in relative terms. RR is calculated as the ratio of the incidence rate of a certain outcome among individuals exposed to a particular factor and the incidence rate among those not exposed to the same risk factor. $\mathrm{OR}$ is calculated as the ratio of the odds that an exposed person develops disease and the odds that an unexposed person develops disease. RR and OR higher than 1 mean that exposure is a risk factor for the outcome. RR and OR lower than 1 mean that exposure is a protective factor for the outcome. RR and OR equal to 1 mean that exposure is not associated with the outcome.
\end{abstract}

Keywords: Incidence among unexposed, Measures of association, Odds ratio, Relative risk

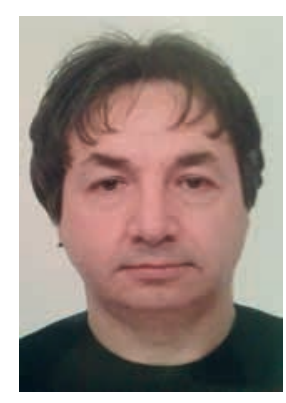

Francesco Franco
Nella precedente pubblicazione dal titolo "Breve guida alla scelta ed interpretazione delle principali misure di associazione utilizzate negli studi clinici" (1) sono state analizzate le misure basate sulla differenza assoluta del rischio (riduzione assoluta del rischio e numero dei casi da trattare per ottenere un esito positivo). In questo numero, invece, si tratteranno le misure basate sulla differenza relativa del rischio, segnatamente il rischio relativo e l'odds ratio.

II rischio relativo e l'odds ratio sono le misure di associazione o di effetto (denominazione, quest'ultima, utilizzata per sottolineare la natura causale di una relazione statistica) più comunemente impiegate in campo epidemiologico (2).

Lo scopo di uno studio epidemiologico è studiare il rapporto causale fra una data esposizione e una specifica malattia (nell'ipotesi che la frequenza della malattia sia diversa

Accepted: November 11, 2017

Published online: November 21, 2017

Indirizzo per la corrispondenza:

franco_francesco@fastwebnet.it

anteo.dinapoli@libero.it tra i soggetti esposti e non esposti al fattore di rischio e che il fattore stesso sia un determinante della patologia) dopo aver escluso l'effetto di altri fattori di rischio. Le misure di associazione basate sulla differenza relativa del rischio, quali il rischio relativo (RR: Relative Risk) e il rapporto fra gli odds (OR: Odds Ratio), forniscono una misura della forza della relazione fra fattore di esposizione e malattia.

II rischio relativo $(R R)$ si ottiene dal rapporto di due tassi di incidenza: il tasso di incidenza di un evento sanitario negli esposti a un fattore di rischio e il tasso di incidenza dello stesso evento sanitario nei non esposti allo stesso fattore di rischio.

$$
R R=\frac{\text { Tasso di inc. }{ }_{(\text {esp.) }}}{\text { Tasso di inc. }{ }_{{ }_{(\text {non esp.) }}}}
$$

Un $R R$ pari ad 1 esprime un uguale tasso di incidenza nel gruppo degli esposti e dei non esposti, riflettendo quindi una mancanza di associazione fra evento ed esposizione poiché il fattore di rischio non influenza la frequenza di occorrenza dell'evento in esame.

Un $R R$ superiore ad 1 , statisticamente significativo, indica la presenza di un'associazione positiva fra evento ed esposizione al fattore di rischio ed esprime di quante volte aumenta il tasso di incidenza negli esposti rispetto a quello dei non esposti. Un rischio relativo pari a 1,7 significa che il rischio di evento nel gruppo degli esposti è maggiore del $70 \%$ del rischio di evento nel gruppo dei non esposti. 
Un $R R$ inferiore ad 1, statisticamente significativo, sta invece $a$ indicare che si è in presenza di un fattore di protezione (associazione negativa, ad esempio vaccinazione) ed esprime di quante volte diminuisce il tasso di incidenza negli esposti (ad esempio, alla vaccinazione) rispetto a quello dei non esposti. Un rischio relativo pari a 0,8 significa che il rischio di evento nel gruppo degli esposti è minore del $20 \%$ del rischio di evento nel gruppo dei non esposti.

Tuttavia, il rischio relativo può essere calcolato correttamente soltanto negli studi longitudinali (di coorte) dove si valuta nel tempo l'incidenza della malattia nel gruppo degli esposti e in quello dei non esposti a un determinato fattore di rischio. Negli studi retrospettivi (caso-controllo), invece, non è possibile la stima dell'incidenza della malattia, essendo sconosciuti sia la dimensione della popolazione degli esposti a rischio di ammalarsi sia il numero complessivo dei nuovi casi occorsi in una determinata finestra temporale (si conosce solo il numero di casi che già si sono verificati).

In tali condizioni è possibile solo calcolare la probabilità per i casi e per i controlli di essere esposti o meno al fattore di rischio in esame. In uno studio retrospettivo, quindi, la misura d'effetto utilizzata è rappresentata dall'odds ratio $(O R)$.

L'odds ratio $(O R)$ è dato dal rapporto di due odds di evento (ad esempio, odds di patologia). L'odds di un evento è dato dal rapporto tra la probabilità $p$ che l'evento si verifichi e la probabilità complementare (1-p) che lo stesso non si verifichi. L'odds ratio, quindi, descrive il rapporto tra gli odds di malattia dato lo stato di esposizione, o in alternativa il rapporto tra gli odds di esposizione data la condizione di malattia (dal punto di vista del calcolo entrambi gli approcci portano allo stesso risultato).

$$
O R=\frac{\frac{p_{(\text {esp. })}}{1-p_{(\text {esp. })}}}{\frac{p_{(\text {non esp. })}}{1-p_{(\text {non esp. })}}}=\frac{\text { odds } s_{(\text {esp. })}}{\text { odds } s_{(\text {non esp. })}}
$$

L'OR non ha un'interpretazione intuitiva come il rischio relativo ed è spesso interpretato come se fosse equivalente al rischio relativo, ignorando il suo significato come rapporto di odds. Un OR pari a 3, ad esempio, viene spesso interpretato in termini di "triplice rischio" di sviluppare una malattia data l'esposizione. Questa inesattezza, potenzialmente, comporta seri errori in quanto l'OR sovrastima sempre il $R R$.

Con alcuni passaggi matematici si può vedere che:

$$
\begin{aligned}
& O R=\frac{\frac{p_{(\text {esp. })}}{1-p_{(\text {esp. })}}}{\frac{p_{(\text {non esp. })}}{1-p_{(\text {non esp. })}}}=\frac{p_{(\text {esp. })}}{1-p_{(\text {esp. })}} * \frac{1-p_{(\text {non esp. })}}{p_{(\text {non esp. })}}= \\
& \frac{p_{(\text {esp. })}}{p_{(\text {non esp. })}} * \frac{1-p_{(\text {non esp. })}}{1-p_{(\text {esp. })}}=R R * \frac{1-p_{(\text {non esp. })}}{1-p_{(\text {esp. })}}
\end{aligned}
$$

dove il fattore di distorsione è rappresentato dalla quantità:

$$
\frac{1-p_{(\text {non esp. })}}{1-p_{(\text {esp. })}}
$$

In presenza di fattori di rischio, la quantità a numeratore del fattore di distorsione è sempre maggiore di quella a denominatore e quindi l'OR sovrastima sempre il $R R$.

TABELLA I - Esempio di calcolo dell'OR e del RR per valori di incidenza della malattia nel gruppo dei non esposti pari al $20 \%$ e al $5 \%$

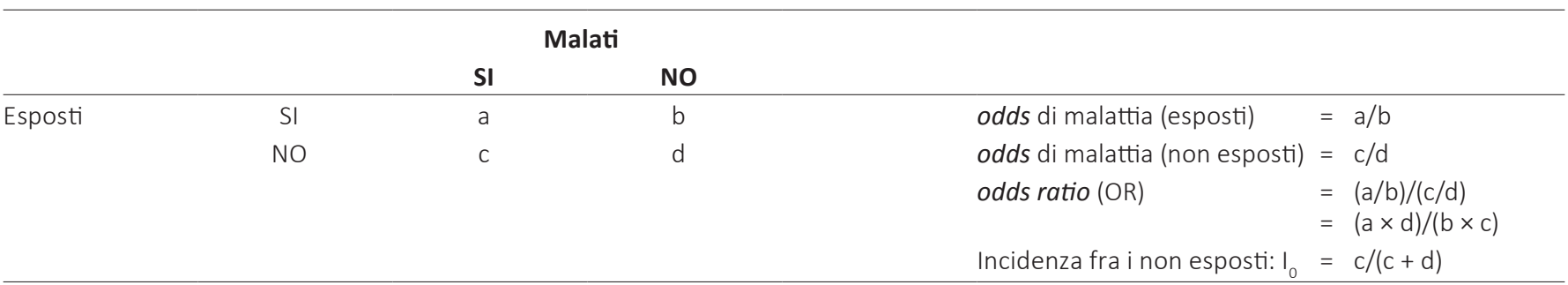

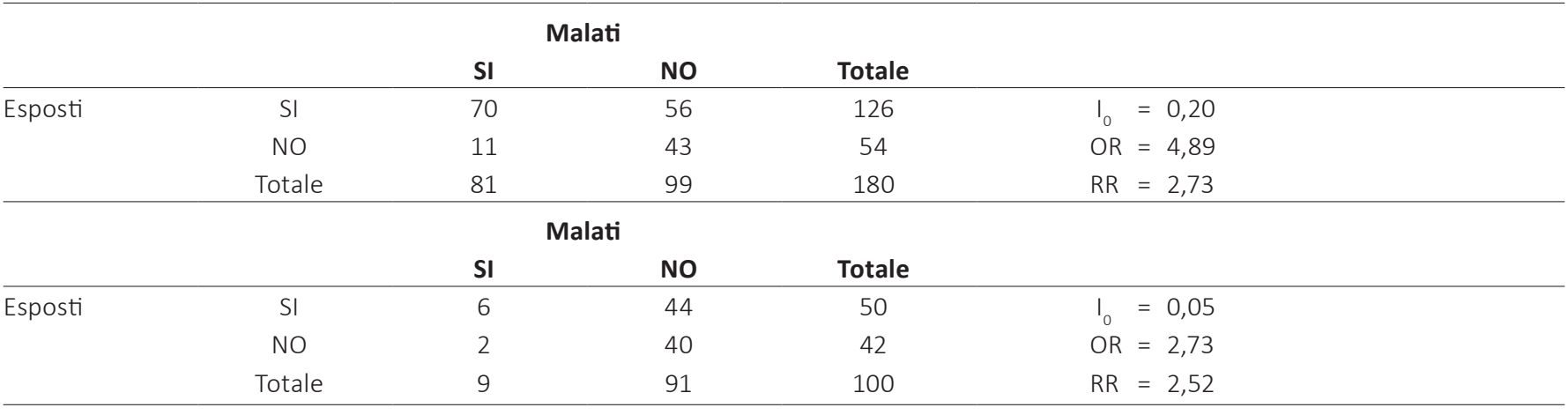


Due sono i fattori che marcano le differenze tra $R R$ e $O R$ : un'alta iniziale incidenza dell'esito di interesse e un'elevata forza dell'associazione fra esposizione ed evento (un $O R$ elevato).

Sotto la cosiddetta "assunzione di malattia rara", I'OR può fornire un'accettabile approssimazione del $R R$. Un'incidenza iniziale dell'evento inferiore al $10 \%$ è considerata essere un cut-off di malattia rara. In alcune situazioni, tuttavia, può essere utile riportare i rischi relativi al posto degli $O R$ per una migliore comunicazione dei risultati anche quando il presupposto di malattia rara non è verificato. Diversi approcci possono essere utilizzati per trasformare un $O R$ in termini di $R R$ fra i quali quella dell'utilizzo di formule di conversione come la seguente (3):

$$
R R=\frac{O R}{\left(1-I_{\text {non esp. }}\right)+\left(I_{\text {non esp. }} * O R\right)}
$$

$L^{\prime} O R$, pur sovrastimando il $R R$ nei casi di alta incidenza della patologia nella popolazione in studio, rimane l'unica preziosa misura utilizzabile per la stima dell'associazione fra esposizione ed evento sanitario negli studi caso-controllo (4).
I vantaggi di tale disegno di studio sono rappresentati da velocità di esecuzione ed economicità dovuti all'utilizzo di basi di dati già esistenti.

Nella Tabella I si riporta un esempio pratico di calcolo dell'OR e del $R R$ per valori di incidenza della malattia nel gruppo dei non esposti pari al $20 \%$ e al $5 \%$.

\section{Disclosures}

Financial support: No financial support was received for this submission.

Conflict of interest: The authors have no conflict of interest.

\section{Bibliografia}

1. Franco F, Di Napoli A. Breve guida alla scelta ed interpretazione delle principali misure di associazione utilizzate negli studi clinici. G Tec Nefrol Dial. 2017;29(3):207-10.

2. Rosmini F. Misure di frequenza e di associazione. In: Mele A, Spada E, Tosti ME, a cura di, Epidemiologia per la clinica e la sanità pubblica. Roma: II Pensiero Scientifico Editore. 2009:18-26.

3. Schmidt CO, Kohlmann T. Int J Public Health. 2008;53:165. https://doi.org/10.1007/ s00038-008-7068-3.

4. Gordis L. Epidemiology. Fourth ed. Philadelphia: Elsevier Saunders. 2014;11:201-4. 